\title{
Ultrasonic-Assisted Thymol and Carvacrol Extraction from Za'atar Leaves Using Cold-Pressed Sesame Oil
}

\author{
Noyal Aweidah, ${ }^{1}$ Hani Naseef $\mathbb{D}^{1},{ }^{1}$ Ramzi Muqdi, ${ }^{1}$ and Mohammad A. Farraj $\mathbb{D}^{2}$ \\ ${ }^{1}$ Department of Pharmacy, Faculty of Pharmacy, Nursing and Health Professions, Birzeit University, P.O Box 14,Birzeit, \\ West Bank, State of Palestine \\ ${ }^{2}$ Faculty of Pharmacy Nursing and Health Professions, Master Program in Clinical Laboratory Science, P.O Box 14,Birzeit, \\ West Bank, State of Palestine \\ Correspondence should be addressed to Hani Naseef; hshtaya@birzeit.edu
}

Received 21 July 2021; Revised 21 September 2021; Accepted 7 October 2021; Published 27 October 2021

Academic Editor: Sobhy El-Sohaimy

Copyright (๑) 2021 Noyal Aweidah et al. This is an open access article distributed under the Creative Commons Attribution License, which permits unrestricted use, distribution, and reproduction in any medium, provided the original work is properly cited.

\begin{abstract}
Thymol and carvacrol found in Origanum syriacum "Za'atar" plant leaves have triggered interest among researchers for their natural antimicrobial and antifungal properties. However, their applications in modern pharmaceutical and cosmetic industries as natural preservatives remain challenging. In this research, extraction of thymol and carvacrol from Za'atar leaves has been investigated using the ultrasonic-assisted method and the use of sesame oil as the primary extraction solvent compared with other vegetable oils including sunflower and olive oils. In addition, a high-performance liquid chromatography (HPLC) analytical method was developed by using a C8 column and $0.05 \%$ trifluoroacetic acid as a buffering agent. The method was validated for specificity, linearity, repeatability, accuracy, and robustness for the analysis of the content of the two terpenes thymol and carvacrol in different Za'atar extracts. Using the validated analytical method, thymol and carvacrol content results revealed the best vegetable oil for extraction. Results showed that sesame oil had the highest content of thymol and carvacrol, followed by sunflower oil, and the least amount obtained by using olive oil. A consistent extraction result opens an opportunity for application in the pharmaceutical and cosmetic industries. For this reason, the essential extraction key parameters that influence the final content of thymol and carvacrol were evaluated. The highest impact originated from the quality and volume of sesame oil. Cold-pressed sesame oil resulted in a 23\% higher content of thymol and decreased amount of carvacrol. It was found that wetting the Za'atar leaves powder with sesame oil was enough for extraction; any additional sesame oil volume dilutes the extracted sample. The second impact was the Za'atar cultivation conditions; less than $10 \%$ difference of thymol and carvacrol content was observed within Za'atar of Hebron origin. Finally, the origin of sesame seeds used for sesame oil production had the least impact.
\end{abstract}

\section{Introduction}

Za'atar is a popular Middle Eastern herb in Arabic culinary. It is considered the most stable and precious herb served in Arabic breakfast meals. The dried leaves are grounded and mixed with sesame seeds, fruits of the tree Rhus coriaria, and salt, where the mixture is served with olive oil and bread $[1,2]$. The herb's leaves are rich in volatile oils that give them a warm, strong aroma that can be described as slightly pungent or acrid. The herb's volatile oils are known to have many therapeutic benefits to which Za'atar is added to tea remedies to treat cold, flu, and cough [3].

Za'atar is classified under the Oregano or Thyme genus and belongs to the plant family Lamiaceae or Labiatae, also known as the mint family. Origanum syriacum var. syriacum is a subspecies of Origanum syriacum that grows about $40 \mathrm{~cm}$ high with simple ovate-shaped green leaves, covered with long and short white hairs and tetragonal and rounded young leaves [2]. This subspecies is classified under the Majorana section found in the Palestinian territories, Jordan, Lebanon, and Syria. The other ten sections of this species that belong to this genus have local distribution across the Mediterranean area [4].

This herb is considered valuable for having a high content of phenolic compounds [3], characterized by having at least one aromatic ring and one hydroxyl group, which gives them antioxidant and antimicrobial powers [5]. Among them, thymol is considered the major monoterpene 
that has antimicrobial activity [6] due to the hydroxyl group at meta position [5]. Carvacrol, an isomer of thymol, has an antimicrobial synergistic effect when combined with thymol [7]. Other terpenes include o-cymene [3] and $\gamma$-terpinene [6]. These four terpenes are the most abundant in Za'atar essential oil [3]. The composition and ratio of Za'atar essential oil components differ according to their geographical distribution or/and the amount of water during plant irrigation [3]. For this reason, wild collection (small production of natural products yearly) can support only a small number of research studies for containing components in a specific ratio that is controlled by the environmental conditions in which it was planted [4]. Za'atar is distributed in many regions in the Palestinian territories including the northern (Nablus, Jenin, and Tulkarem), southern (Hebron), and central (Ramallah and Bethlehem) areas. Za'atar in these areas has different oil compositions and different yields [3]. Indeed, soil composition and percentage of humidity differ from one area to another, which makes cultivation important for large-scale production [4], where thymol production can be induced by selection of a vegetable compost soil [8] and water irrigation [3].

Since thymol and carvacrol are the major phenolic components of the plant, which are considered active constituents, it is important to develop a valid method for the assay of these compounds. High-performance liquid chromatography (HPLC) is a popular method for the analysis of herbal components. HPLC is one of the most precise techniques for the quantitative determination of plant constituents, which is used for volatile and nonvolatile compounds [9]. HPLC was used successfully for the quality assessment of thymol and carvacrol in previous research studies [10-12].

Extraction technologies have been developed through time where some are considered classical and others advanced methods. Steam distillation is one of the classical extraction methods that was introduced long ago by the Arabs, where all the plant's components are extracted [13]. Solid-to-liquid extractions are selective methods where the extraction solvent and solute have similar polarities. In these types of extractions, different technologies are applied, such as supercritical fluid extraction, pressurized liquid extraction (PLE) and/or temperature "accelerated solvent extraction" (ASE) [14], ultrasonic assistance, and microwave assistance or enzymatic assistance [5].

Nowadays, great importance has been paid to using green extraction methods [15]. Green solvents that do not harm humans and the environment are highly recommended for use instead of hazardous solvents such as the widely used hexane. Such a replacement is based on finding a green solvent that has similar polarity and basicity qualities to the hazardous one [16].

In 2017, Mnayer published a green solid-to-liquid ultrasonic extraction method. The study compared conventional extraction techniques using hexane and ethanol solvents versus green solvent (sunflower oil) and absolute ethanol. Mnayer also compared extraction with and without the aid of ultrasonication at different temperatures in the range of $10^{\circ} \mathrm{C}$ to $50^{\circ} \mathrm{C}$, sonication time in the range of 7 to 23 minutes, and ultrasonic power in the range of 32 to $98 \mathrm{~W}$. A better quality yield and more content of thymol were achieved by extraction with sunflower oil solvent using the optimized parameters of $50.18^{\circ} \mathrm{C}$ for sonication temperature, 22 minutes for sonication time, and $98.63 \mathrm{~W}$ for ultrasonication power [17].

In this study, thymol and carvacrol extraction from Hebron Za'atar leaves was performed by using the ultrasonic-assisted method and the use of sesame oil as an alternative green, nontoxic natural solvent. Results were compared with those of other vegetable oils including sunflower and olive oils. In addition, a new HPLC method was developed and validated for the analysis of thymol and carvacrol content in extracted samples. Finally, a comparison study of thymol and carvacrol content in different extract samples was performed to determine the extraction key parameters.

\section{Materials and Methods}

\subsection{Instrument and Materials}

2.1.1. For Analytical Method Development. The equipment used is as follows: HPLC from the Agilent 1200 model with UV double-beam PerkinElmer model, UV spectrophotometer, ultrasonic bath $2 \mathrm{~L}$ with a frequency of $28 / 40 \mathrm{kHz}$ and ultrasonic power of $120 \mathrm{~W}$, pH meter, analytical balance, and semianalytical balance.

The chemicals used are as follows: phosphoric acid HPLC grade from J. T. Baker, catalog number 02-003-613; trifluoracetic acid HPLC grade from Sigma-Aldrich, catalog number 302031; acetonitrile 99.9\% HPLC grade from Fisher Scientific, catalog number A998-212; thymol standard from Acros Organics, catalog number 15033; carvacrol standard from Sigma-Aldrich, catalog number 282197; and column C8 $150 \times 4.6 \mathrm{~mm}$ from Restek, catalog number 9533565 .

2.1.2. For Extraction. The equipment and materials used are as follows: ultrasonic bath $2 \mathrm{~L}$ with a frequency of $28 / 40 \mathrm{kHz}$ and ultrasonic power of $120 \mathrm{~W}$; thermometer; semianalytical balance; cold press oil machine; magnetic stirrer $5 \mathrm{~L}$; retroevaporator; olive oil, sunflower oil, sesame oil, all three from Iz Adin company; Whatman filter; sesame seeds; and absolute ethanol 99.8\% from Sigma-Aldrich, catalog number 24102.

\subsection{Method Development}

2.2.1. UV Selection. The absorbance of thymol and carvacrol was measured separately using UV-visible spectrophotometry over the range of $200 \mathrm{~nm}$ to $400 \mathrm{~nm}$.

2.2.2. Selection of Mobile Phase and Column. The initial mobile phase and column were selected according to Shekarchi method [18]. Injections were started using C18, $250 \mathrm{~mm} \times 4.6 \mathrm{~mm}$, and a $5 \mu \mathrm{m}$ column. Isocratic mobile phase combination of $50: 50$ (ACN : PW), flow rate of $1 \mathrm{ml} /$ min, injection volume of $10 \mu \mathrm{l}$, diluent of $80: 20$, and ratio of ACN : PW. For buffering the system, a buffering agent was 
added to the mobile phase to improve the tailing and peak shapes by testing different concentrations of $0.5 \%$ or $3 \%$ diluted phosphoric acid. After several trials, it was observed that the tailing of peaks cannot be improved as the C-18 column was not suitable for the low $\mathrm{pH}$ range. To resolve this issue, a C8 column was used instead for having lower hydrophobicity and for separating nonpolar organics, where only a small percentage of trifluoracetic acid (TFA) (0.05\%) was used.

2.2.3. Selected Parameters for Analysis. Column: C8 $150 \mathrm{~mm} \times 4.6 \mathrm{~mm}, 5 \mu \mathrm{m}$ column; flow rate: $1.0 \mathrm{ml} / \mathrm{min}$; UV: $205 \mathrm{~nm}$; injection vol: $10 \mu \mathrm{l}$; column temperature: $30^{\circ} \mathrm{C}$; mobile phase A: $50 \%(\mathrm{ACN})$; and mobile phase $\mathrm{B}: 50 \%$ (0.05\% TFA buffer).

2.2.4. Validation of Analytical Method. The developed method was tested for specificity, linearity, accuracy, repeatability, and robustness for thymol and carvacrol.

\subsection{Extraction}

2.3.1. Collection of Za'atar Plant Leaves. Fresh leaves of Za'atar were obtained from two different regions in Hebron. The leaves were cut from the stem and dried for 14 days using a sieve; then, they were stored in a plastic bag in the dark.

2.3.2. Solid-to-Liquid Extraction Using Green Solvents (Olive, Sunflower Oil, and Sesame Oil). Plant material was milled using a coffee mill machine. One gram of milled material was combined with each of the solvents separately (sunflower oil, olive oil, or sesame oil) in a $100 \mathrm{ml}$ volumetric flask. The mixture was sonicated at $50^{\circ} \mathrm{C}$ for 25 minutes with agitation every 7 minutes. After sonication, the volumetric flask was cooled and filled to volume with the solvent and was shaken well.

The mixture was filtered with a coffee filter and transferred to a beaker where absolute ethanol was added in a ratio of 1:1.5 (mixture: absolute ethanol) and stirred well. The mixture was transferred to a separating funnel and was agitated vigorously and allowed to stand for one hour. The supernatant was evaporated at $40^{\circ} \mathrm{C}$. The yield was collected, filtered using a Buchner funnel vacuum flask, filtered by a Whatman filter paper, and stored in the refrigerator at $5^{\circ} \mathrm{C}$.

2.3.3. Cold-Pressed Sesame Oil Production. Sesame seeds were pressed using an oil pressing machine where the temperature was monitored and maintained below $45^{\circ} \mathrm{C}$ during the whole process. Low temperatures protect the oil components from degradation [19]. Sesame seeds from two different origins, Turkey and India, were pressed separately through a single screwdriver. The oil is collected from underneath the screwdriver, and the peels of sesame are expelled from the other side. Sesame oil was filtered using a coffee filter and left for 24 hours. The upper yellow part was collected, where the white part was put in a small jar for further precipitation. Sesame oil was stored in a brown bottle.

2.3.4. Solid-to-Liquid Extraction Using Sesame Oil Scale-Up. Thirty grams of dried plant material were milled using a coffee mill machine. The milled material was weighed and transferred to a $2000 \mathrm{ml}$ beaker, and $350 \mathrm{ml}$ of sesame oil was added. The beaker was sonicated at $50^{\circ} \mathrm{C}$ for 25 minutes with agitation every 7 minutes. The mixture was filtered with a coffee filter and transferred to a beaker where absolute ethanol was added in a ratio of 1:1.5 (mixture: absolute ethanol) and stirred for 15 minutes using a magnetic stirrer. The supernatant was collected, and the rest of the oil was transferred into a separating Buchner funnel to separate the remaining alcohol and allowed to stand for 1 hour. The supernatant was added to the collected alcohol and was evaporated at $40^{\circ} \mathrm{C}$. The yield was collected, filtered using Whatman filter paper, and stored in the fridge at $5^{\circ} \mathrm{C}$.

2.3.5. Analysis of Za'atar Extracts. The extraction method of thymol and carvacrol from Za'atar leaves was evaluated for quality and consistency of the extracted material. A comparison study using analysis of Za'atar extracts by the validated HPLC method was applied. The purpose of this study was to determine the content of thymol and carvacrol and limit the content variation in a raw material batch by identifying the responsible factors. The study covered the following issues.

2.3.6. Variations in Za'atar Extracts. Samples of Za'atar were obtained from two different areas in Hebron. In this study, these samples are referred to as Hebron Za'atar A and Hebron Za'atar B. They were extracted using the scale-up method, by using the lab-made sesame oil using a cold press machine.

2.3.7. Variations in Sesame Oil. Hebron Za'atar A was extracted from two different sesame oils: the first oil used was Izz-Aldin sesame oil, and the second oil was the labmade sesame oil using a cold-press machine.

2.3.8. Variations in Sesame Seed Origin. Hebron Za'atar A sample quantity was small; therefore, the study was continued using Hebron Za'atar B.

Hebron Za'atar B was extracted using two different sesame oils made of different origins of sesame seeds (Indian and Turkish).

2.3.9. Sample Preparation for HPLC Analysis. One (1.0) gram of extracted material was weighed into a $50 \mathrm{ml} \mathrm{VF}$ and made up to volume with diluent (ACN $80: \mathrm{PW} 20)$ and sonicated at room temperature for 10 minutes. Five (5.0) $\mathrm{ml}$ of prepared sample was diluted to a $25 \mathrm{ml} \mathrm{VF}$ with the same diluent. All samples were injected into HPLC using the validated method. 
2.3.10. Extraction Optimization. By using the scale-up extraction method, Hebron Za'atar B was extracted by using sesame oil made by cold pressing of Indian sesame seeds. The volume of sesame oil was decreased from $350 \mathrm{ml}$ to $250 \mathrm{ml}$, where Za'atar powder was all wetted with the solvent.

\section{Results}

3.1. UV Scanning. Thymol and carvacrol both showed high absorbance at 204 and $270 \mathrm{~nm}$, as shown in Figure 1. Thus, in method development, $204 \mathrm{~nm}$ was used as the absorbance wavelength.

3.2. Selection of Column and Mobile Phase. The selection of column and mobile phase was started by using a C18 column and isocratic mobile phase containing $0.5 \%$ diluted phosphoric acid and 50\% ACN. The peaks were not ideal, and a resolution of less than 2 was achieved between carvacrol and thymol. On the same hand, the method had bad tailing for thymol and carvacrol when the phosphoric acid was increased to $3 \%$. After switching to column C8, using $0.05 \%$ of TFA as a buffering agent and $50 \% \mathrm{ACN}$, tailing and resolution were achieved with ideal peaks for both compounds in two different concentrations of carvacrol and thymol $(0.01 \mathrm{mg} / \mathrm{ml}$ and $0.1 \mathrm{mg} / \mathrm{ml}$, respectively), as shown in Figures 2(a) and 2(b).

3.3. Validation of Analytical Method. The analytical method was successfully validated to be used for the analysis of thymol and carvacrol in a raw material sample. First, the method was found to be specific, as there was no mobile phase or diluent peaks interfering under the major peaks of carvacrol and thymol. Second, it was found to be linear in the range of 0.001 to $0.12 \mathrm{mg} / \mathrm{ml}$ for both thymol with a coefficient of 0.9995 and carvacrol with a coefficient of 0.9996 . Third, the method obtained 0.5\% RSD for both thymol and carvacrol, exhibiting the method's repeatability. Fourth, the method was found to be accurate as the mean recovery for thymol was $101.6 \%$ and carvacrol was $100.8 \%$. Finally, the method was robust as it met the system suitability under different conditions.

3.4. Results of Extraction Using Different Oils. It was found that sesame oil has the highest amount of thymol and carvacrol, followed by sunflower oil, and the lowest amount obtained by using olive oil, as shown in Table 1 . This makes sesame oil $42 \%$ more efficient in extraction than sunflower oil.

\subsection{Analysis of Za'atar Extracts' Results}

3.5.1. Results of Different Za'atar Extracts. As shown in Table 2, it is obvious that there was a small difference in the quantities of thymol obtained from Za'atar extracts according to the area.

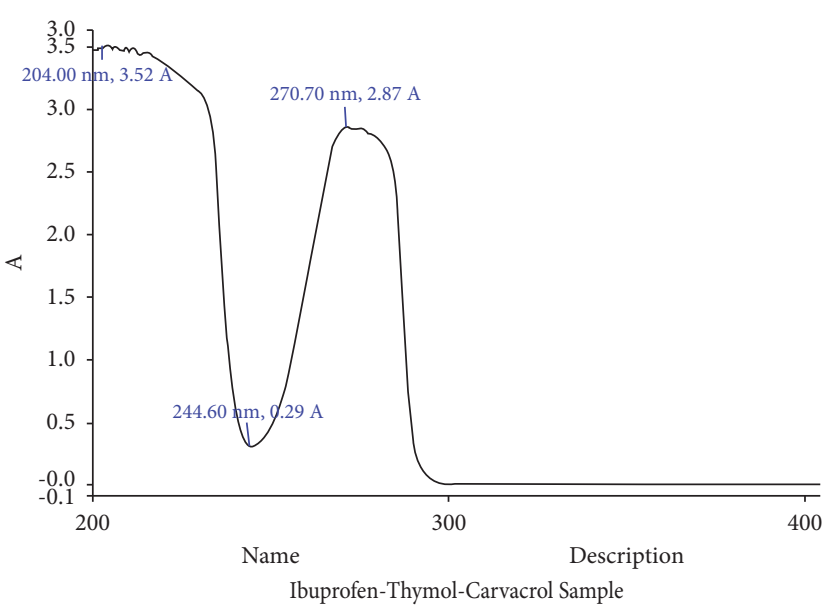

Figure 1: Absorbance area for thymol and carvacrol. Thymol and carvacrol standards were measured over the range of $200 \mathrm{~nm}$ to $400 \mathrm{~nm}$.

3.5.2. Results of Za'atar "A" Extract by Using Different Sesame Oils. As shown in Table 3, a 23\% higher amount of thymol was detected when cold-pressed sesame oil was used versus commercial sesame oil. Contrary to that, the amount of carvacrol was reduced by $36 \%$ when cold-pressed sesame oil was used.

3.5.3. Results of Hebron Za'atar Using Sesame Oil from Different Seed Origins. As shown in Table 4, slightly higher amounts of thymol and carvacrol were detected by using the Indian sesame seeds.

3.5.4. Results of Reducing Sesame Oil Volume. As shown in Table 5, the content of thymol is higher and lower yields of thymol and carvacrol were found by reducing the volume of sesame oil, resulting in a more concentrated extract.

\section{Discussion}

Several vegetable oils have been used as cosolvents for extraction. The advantages of the addition of vegetable oils as cosolvents have been illustrated to enhance the yield depending on the physicochemical properties of the used oil [20-23]. In the present study, the selected oils for comparison are widely available in the Palestinian market. Sesame oil-absolute ethanol had a very surprising result as it was $42 \%$ more efficient than sunflower oil-absolute ethanol for both thymol and carvacrol, whereas olive oil yield of thymol and carvacrol was minimal.

The scale-up extraction method increased the content of thymol and carvacrol. This difference could be explained by gaining more extraction practice with time, extracting larger samples of Za'atar leaves, using larger equipment, and using a magnetic stirrer for longer sesame oil-absolute ethanol mixing time.

It can be found that for each 100-gram plant leaves, Mnayer extraction yielded 5.93 grams of oil, which contained $171.22 \mathrm{mg} / \mathrm{g}$ of total phenolic content comprising 
VWD1A, Wavelength $=205 \mathrm{~nm}$

(IBUPROFEN- THYMOL- CARVACROL 2021-05-04 15-48-461071-1802.D)

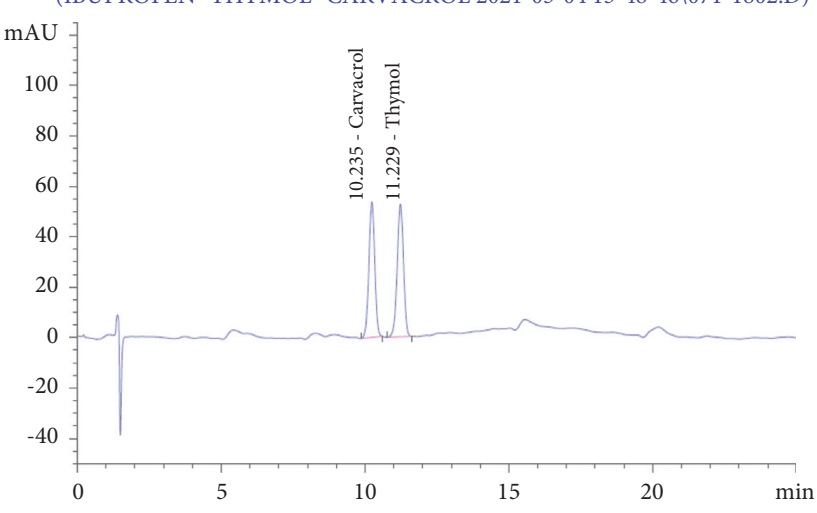

(a)
VWD1 A, Wavelength $=205 \mathrm{~nm}$

(IBUPROFEN- THYMOL- CARVACROL 2021-04-06 14-38-25\010-0901.D)

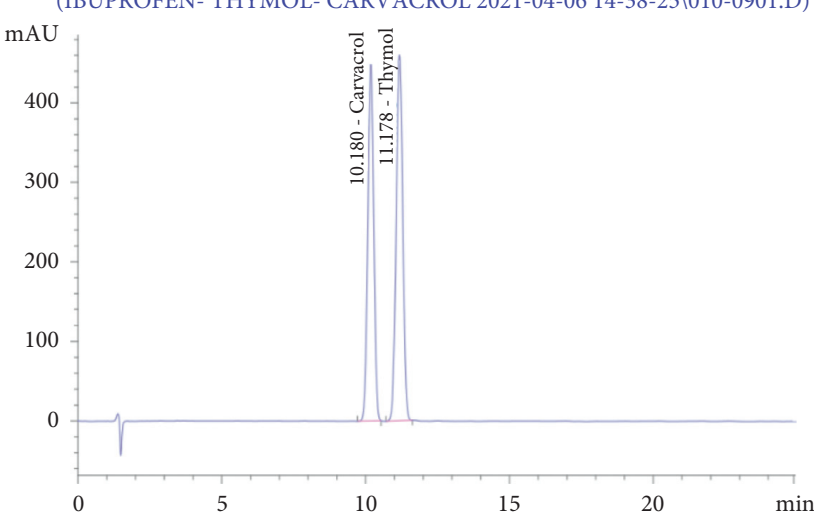

(b)

Figure 2: Chromatograms of thymol and carvacrol standards. $0.01 \mathrm{mg} / \mathrm{ml}$ thymol and carvacrol standards (a) and $0.1 \mathrm{mg} / \mathrm{ml}$ thymol and carvacrol (b) were injected to HPLC using the developed method.

TABle 1: Thymol and carvacrol content in Za'atar extracts using olive oil, sunflower oil, and sesame oil for extraction.

\begin{tabular}{lcc}
\hline Oil used for extraction & Carvacrol $(\mathrm{mg}) / 1$ gram & Thymol (mg)/1 gram \\
\hline Olive oil & 0.35 & 0.305 \\
Sunflower oil & 1.6 & 8.15 \\
Sesame oil & 1.85 & 14.11 \\
\hline
\end{tabular}

TABLE 2: Carvacrol and thymol content in different locations of Hebron Za'atar.

\begin{tabular}{lcc}
\hline Sample name & Carvacrol $(\mathrm{mg}) / 1 \mathrm{gram}$ & Thymol (mg)/1 gram \\
\hline Hebron Za'atar A extract & 3.57 & 25.71 \\
Hebron Za'atar B extract & 1.33 & 23.40 \\
\hline
\end{tabular}

TABLE 3: Carvacrol and thymol content by using regular and cold-pressed sesame oil.

\begin{tabular}{lcc}
\hline Sample name & Carvacrol (mg)/1 gram & Thymol (mg)/1 gram \\
\hline Hebron Za'atar A extract by using cold-pressed sesame oil at lab & 3.57 & 25.71 \\
Hebron Za'atar A extract by using Izz-Aldin sesame oil & 5.59 & 19.83 \\
\hline
\end{tabular}

TABLE 4: Carvacrol and thymol content by using different origins of sesame seeds.

\begin{tabular}{lcc}
\hline Sample name & Carvacrol $(\mathrm{mg}) / 1$ gram & Thymol (mg)/1 gram \\
\hline Hebron Za'atar B, sesame oil from Indian sesame & 2.5 & 22.0 \\
Hebron Za'atar B, sesame oil from Turkish seeds & 2.2 & 21.2 \\
\hline
\end{tabular}

TABLE 5: Thymol and carvacrol content and yield when the volume of sesame oil is reduced.

\begin{tabular}{lccc}
\hline Sample name & Carvacrol $(\mathrm{mg}) / 1$ gram & Thymol $(\mathrm{mg}) / 1$ gram & Yield of oil $(\mathrm{ml})$ \\
\hline Hebron Za'atar B by using $250 \mathrm{ml}$ sesame oil & 4.4 & 32.8 & 14 \\
Hebron Za'atar B by using $350 \mathrm{ml}$ sesame oil & 2.5 & 22.0 & 20 \\
\hline
\end{tabular}

$80.5 \%$ of thymol and $5.7 \%$ of carvacrol. The Mnayer method resulted in $817.34 \mathrm{mg}$ of thymol and $57.87 \mathrm{mg}$ of carvacrol. However, for each 100-gram plant extracted by the scaled-up sesame oil-absolute ethanol method, it yielded 66.66 grams of oil, which contained $23.4 \mathrm{mg} / \mathrm{g}$ of thymol and $1.33 \mathrm{mg} / \mathrm{g}$ of carvacrol, resulting in $1559.84 \mathrm{mg}$ of thymol and $88.66 \mathrm{mg}$ of carvacrol. The cold-pressed sesame oil-absolute ethanol extraction is $50.2 \%$ more efficient than the Mnayer 
extraction of sunflower oil-absolute ethanol extraction. This finding makes cold-pressed sesame oil-absolute ethanol the best green solvent combination for the extraction of thymol and carvacrol using the ultrasonic method.

A comparison study of thymol extraction from Thymus vulgaris was performed between green solvent ethanol, limonene, and ethyl lactate by pressurized liquid extraction (PLE) and supercritical fluid extraction (SFE) by Bermejo. The highest thymol content achieved in this study was between 7 and $11 \mathrm{mg}$ of thymol for 1 gram by the SFE method [24], whereas the ultrasonic method using sesame oil-absolute ethanol achieved 20-25 mg thymol in 1-gram dried leaves. By using the ultrasonic method, a double thymol content was achieved as compared to the expensive SFE and PLE methods.

The extracted Za'atar oil can be used as a natural preservative in the pharmaceutical and cosmetic industries [25]. Extraction methods must be fully understood to produce consistent batches that have minimum variations. The most important parameter in extraction consistency is the quality and volume of sesame oil. Depending on the method of pressing, commercial sesame oil quality can differ from one vendor to another [26,27]. It was found that higher quality sesame oil where sesame seeds were milled under $45^{\circ} \mathrm{C}$ resulted in a $23 \%$ higher thymol content than the regular sesame oil. The optimal volume of sesame oil required for extraction was determined by reducing the volume of sesame oil. The minimum volume of sesame oil of $250 \mathrm{ml}$ is needed to wet 30 grams of milled Za'atar powder where it resulted in less yield of Za'atar extract oil but more concentrated with thymol content.

Plant leaves origin and cultivation conditions are other parameters affecting the consistency of extraction. Hebron Za'atar origin was selected as it has the biggest share in the Palestinian market. The Za'atar plant leaf with an acrid taste is an indicator for containing thymol. By selecting only bitter Za'atar leaves from the Hebron area, less than a $10 \%$ difference was found in thymol content, which makes it a reasonably accepted criterion.

Finally, it was found that the origin of sesame seeds used for pressing has a minor effect on extraction consistency. By comparing the two major sources of sesame seeds that reach Palestinian territories, the Turkish and Indian sesame seeds, the Indian sesame seeds resulted in slightly higher thymol and carvacrol content than the Turkish sesame seeds.

\section{Conclusion}

The combination of using ultrasonic and sesame oil-absolute ethanol solvent for the extraction of thymol and carvacrol can be described as an easy, inexpensive, and efficient method. The method used by Mnayer where ultrasonic and sunflower oil-absolute ethanol had almost the same result of using a conventional extraction method with hexane. The use of sesame oil instead of sunflower oil increases the extraction efficiency by $50.2 \%$. This makes the method very efficient and can definitely replace the use of conventional extraction by hexane which is also better for the environment and safer for humans.

\section{Data Availability}

The data used to support the findings of this study are available from the corresponding author upon request.

\section{Conflicts of Interest}

The authors declare that they have no conflicts of interest.

\section{Acknowledgments}

The authors acknowledge the Department of Pharmacy and the Faculty of Pharmacy, Nursing and Health Professions at Birzeit University for their support in conducting this research.

\section{References}

[1] R. A. Alwafa, S. Mudalal, and G. Mauriello, "Origanum syriacum L. (Za'atar), from raw to go: a review," Plants, vol. 10, no. 5 , p. 1001, 2021.

[2] S. O. Saad Bashar, Greco-Arab and Islamic Herbal Medicine Traditional System, Ethics, Safety, Efficacy, and Regulatory Issues, John Wiley \& Sons, Inc., Hoboken, New Jersey, 2011.

[3] S. Abu-Lafi, I. Odeh, H. Dewik, M. Qabajah, L. O. Hanuš, and V. M. Dembitsky, "Thymol and carvacrol production from leaves of wild Palestinian Majorana syriaca," Bioresource Technology, vol. 99, no. 9, pp. 3914-3918, 2008.

[4] F. Thonner and R. Geesink, Thonner's Analytical Key to the Families of Flowering Plants, Centre for Agricultural Publishing and Documentation, Wageningen, Netherlands, 1981.

[5] M. M. Beya, M. E. Netzel, Y. Sultanbawa, H. Smyth, and L. C. Hoffman, "Plant-based phenolic molecules as natural preservatives in comminuted meats: a review," Antioxidants, vol. 10, no. 2, p. 263, 2021.

[6] M. Shehadeh, N. Jaradat, M. Al-Masri et al., "Rapid, costeffective and organic solvent-free production of biologically active essential oil from Mediterranean wild Origanum syriacum," Saudi Pharmaceutical Journal, vol. 27, no. 5, pp. 612-618, 2019.

[7] S. Chouhan, K. Sharma, and S. Guleria, "Antimicrobial activity of some essential oils-present status and future perspectives," Medicine, vol. 4, no. 3, p. 58, 2017.

[8] I. El-Alam, R. Zgheib, M. Iriti et al., "Origanum syriacum essential oil chemical polymorphism according to soil type," Foods, vol. 8, no. 3, p. 90, 2019.

[9] R. Govindarajan, V. Tejas, and P. Pushpangadan, "Highperformance liquid chromatography (HPLC) as a tool for standardization of complex herbal Drugs," Journal of AOAC International, vol. 102, no. 4, pp. 986-992, 2019.

[10] T. Angelo, F. Q. Pires, G. M. Gelfuso, J. K. R. da Silva, T. Gratieri, and M. S. S. Cunha-Filho, "Development and validation of a selective HPLC-UV method for thymol determination in skin permeation experiments," Journal of Chromatography B, vol. 1022, pp. 81-86, 2016.

[11] M. Ghaedi, M. Roosta, S. Khodadoust, and A. Daneshfar, "Application of optimized vortex-assisted surfactant-enhanced DLLME for preconcentration of thymol and carvacrol, and their determination by HPLC-UV: response surface methodology," Journal of Chromatographic Science, vol. 53, no. 7, pp. 1222-1231, 2015.

[12] L. I. Alekseeva, "Determining thymol and carvacrol by reversed-phase high-performance liquid chromatography," 
Pharmaceutical Chemistry Journal, vol. 43, no. 12, pp. 665667, 2009.

[13] K. H. Başer and G. Buchbauer, Handbook of Essential Oils: Science, Technology, and Applications, CRC Press, Boca Raton, 2010.

[14] S. C. Mandal, V. Mandal, and A. K. Das, "Classification of Extraction Methods," Essentials of Botanical Extraction, pp. 83-136, Elsevier, 1st edition, 2015.

[15] P. Panja, "Green extraction methods of food polyphenols from vegetable materials," Current Opinion in Food Science, vol. 23, pp. 173-182, 2018.

[16] S. P. J. Kumar, S. R. Prasad, R. Banerjee, D. K. Agarwal, K. S. Kulkarni, and K. V. Ramesh, "Green solvents and technologies for oil extraction from oilseeds," Chemistry Central Journal, vol. 11, no. 1, 2017.

[17] D. Mnayer, A.-S. Fabiano-Tixier, E. Petitcolas, K. Ruiz, T. Hamieh, and F. Chemat, "Extraction of green absolute from thyme using ultrasound and sunflower oil," Resource-Efficient Technologies, vol. 3, no. 1, pp. 12-21, 2017.

[18] M. Shekarchi, M. Khanavi, N. Adib, M. Amri, and H. Hajimehdipoor, "A validated high performance liquid chromatography method for the analysis of thymol and carvacrol in Thymus vulgaris L. volatile oil," Pharmacognosy Magazine, vol. 6, no. 23, p. 154, 2010.

[19] M. Imran, M. K. Khan, M. Ali et al., "Cold pressed sesame (Sesamum indicum) oil," Cold Pressed Oils, , pp. 105-111, Elsevier, 2020.

[20] E. Yara-Varón, Y. Li, M. Balcells, R. Canela-Garayoa, A.-S. Fabiano-Tixier, and F. Chemat, "Vegetable oils as alternative solvents for green oleo-extraction, purification and formulation of food and natural products," Molecules, vol. 22, no. 9, p. 1474, 2017.

[21] M. Sun and F. Temelli, "Supercritical carbon dioxide extraction of carotenoids from carrot using canola oil as a continuous co-solvent," The Journal of Supercritical Fluids, vol. 37, no. 3, pp. 397-408, 2006.

[22] J. L. Luque-Garćia and M. D. Luque de Castro, "Ultrasoundassisted Soxhlet extraction: an expeditive approach for solid sample treatment," Journal of Chromatography A, vol. 1034, no. 1-2, pp. 237-242, 2004.

[23] G. Vasapollo, L. Longo, L. Rescio, and L. Ciurlia, "Innovative supercritical CO2 extraction of lycopene from tomato in the presence of vegetable oil as co-solvent," The Journal of Supercritical Fluids, vol. 29, no. 1-2, 2004.

[24] D. Villanueva Bermejo, I. Angelov, G. Vicente et al., "Extraction of thymol from different varieties of thyme plants using green solvents," Journal of the Science of Food and Agriculture, vol. 95, no. 14, pp. 2901-2907, 2015.

[25] A. Hassoun, M. Carpena, M. A. Prieto et al., "Use of spectroscopic techniques to monitor changes in food quality during application of natural preservatives: a review," Antioxidants, vol. 9, no. 9, p. 882, 2020.

[26] M. G. Agidew, A. A. Dubale, M. Atlabachew, and W. Abebe, "Fatty acid composition, total phenolic contents and antioxidant activity of white and black sesame seed varieties from different localities of Ethiopia," Chemical and Biological Technologies in Agriculture, vol. 8, no. 1, 2021.

[27] J. Mistrello, S. D. Sirisena, A. Ghavami, R. J. Marshall, and S. Krishnamoorthy, "Determination of the antioxidant capacity, total phenolic and flavonoid contents of seeds from three commercial varieties of culinary dates," International Journal of Food Studies, vol. 3, no. 1, 2014. 